\title{
Succinylated Gelatin Improves the Theranostic Potential of Radiolabeled Exendin-4 in Insulinoma Patients
}

\author{
Mijke Buitinga ${ }^{1}$, Tom Jansen ${ }^{1}$, Inge van der Kroon ${ }^{1}$, Wietske Woliner-van der Weg ${ }^{1}$, Marti Boss ${ }^{1}$, Marcel Janssen ${ }^{1}$, \\ Erik Aarntzen ${ }^{1}$, Martin Béhé ${ }^{2}$, Damian Wild ${ }^{3}$, Eric Visser ${ }^{1}$, Maarten Brom ${ }^{1}$, and Martin Gotthardt ${ }^{1}$ \\ ${ }^{I}$ Department of Radiology and Nuclear Medicine, Radboud University Medical Center, Nijmegen, The Netherlands; ${ }^{2}$ Center for \\ Radiopharmaceutical Sciences, ETH-PSI-USZ, Paul Scherrer Institute, Villigen, Switzerland; and ${ }^{3}$ Division of Nuclear Medicine, \\ University Hospital Basel, Basel, Switzerland
}

\begin{abstract}
Being highly expressed in insulinomas, the glucagonlike peptide-1 receptor $(\mathrm{GLP}-1 \mathrm{R})$ is a potential target for diagnosis, localization, and treatment with the radiolabeled GLP-1R agonist exendin. Tracer accumulation in the kidneys, however, hampers accurate diagnostic visualization of pancreatic tissue and prohibits the therapeutic application of radiolabeled exendin for $\beta$-cell-derived tumors. Therefore, we evaluated the ability of succinylated gelatin (Gelofusine) to reduce the renal accumulation of radiolabeled exendin in humans, and we performed dosimetric calculations to estimate the maximum absorbed insulinoma dose that could be achieved if exendin were to be used for peptide receptor radionuclide therapy. Methods: Ten healthy volunteers received $50 \mathrm{MBq}$ of ${ }^{111}$ In-exendin-4, in combination with Gelofusine or saline, in a crossover design. SPECT/CT images were obtained after $24 \mathrm{~h}$. The procedure was repeated $3 \mathrm{wk}$ later. Uptake of ${ }^{111} \mathrm{In}$-exendin was determined by drawing regions of interest around the kidneys and in the pancreas. Planar scintigraphic ${ }^{111} \mathrm{In}$-exendin images of $5 \mathrm{insu}-$ linoma patients were used for dosimetry studies estimating the maximum insulinoma absorbed dose that could be achieved without causing radiotoxicity to other organs. Results: Gelofusine reduced the renal accumulation of ${ }^{111}$ In-exendin- 4 by $18.1 \%$, whereas the pancreatic uptake remained unchanged. In 3 of 10 subjects, the kidney uptake was reduced to such an extent that the pancreatic tail could be better discriminated from the kidney signal. Dosimetric estimations suggested that the insulinoma absorbed dose ranges from 30.3 to 127.8 Gy. This dose could be further increased to maximally 156.1 Gy if Gelofusine was used. Conclusion: We have shown that Gelofusine can reduce the renal accumulation of ${ }^{111} \mathrm{In}$-exendin-4 in humans. This reduction not only allows more accurate qualitative and quantitative analyses of radiolabeled exendin uptake in the tail region of the pancreas but also potentiates the safe delivery of a higher radiation dose to GLP-1Rpositive tumors for therapy.
\end{abstract}

Key Words: exendin-4; kidney uptake; insulinoma; radionuclide therapy; dosimetry

J Nucl Med 2019; 60:812-816

DOI: 10.2967/jnumed.118.219980

\footnotetext{
Received Sep. 6, 2018; revision accepted Oct. 26, 2018.

For correspondence or reprints contact: Martin Gotthardt, Department of Radiology and Nuclear Medicine, Radboud University Medical Center, P.O. Box 9101, 6500 HB Nijmegen, The Netherlands.

E-mail: Martin.Gotthardt@radboudumc.nl

Published online Nov. 30, 2018.

COPYRIGHT (C 2019 by the Society of Nuclear Medicine and Molecular Imaging.
}

I nsulinomas are rare neuroendocrine tumors arising from pancreatic $\beta$-cells (1). Most patients with insulinomas experience hypoglycemic events due to uncontrolled insulin secretion. To date, surgery is the only curative treatment. However, in approximately $10 \%$ of the cases, patients have multiple lesions or metastatic disease, hampering successful surgical intervention (2). Therefore, preoperative localization of the lesions is critical.

Since insulinomas are typically very small $(<1 \mathrm{~cm})$, conventional imaging methods such as MRI, CT, and endoscopic ultrasound often experience difficulties in exactly localizing the smallest lesions (3). Through the development of radiolabeled peptide ligands, such as somatostatin analogs, nuclear imaging techniques have now become available to detect small insulinoma lesions with a higher sensitivity than conventional techniques (4-7). Diagnosis can be followed by personalized treatment using these analogs, labeled with, for example, ${ }^{90} \mathrm{Y}$ or ${ }^{177} \mathrm{Lu}(8)$. Encouraging clinical results (8-11) advocate somatostatin peptide receptor radionuclide therapy (PRRT) as an alternative treatment strategy for patients with unresectable or metastatic insulinomas $(10,12,13)$.

However, in about $40 \%$ of the benign and $30 \%$ of the malignant insulinomas, the expression of the somatostatin receptor is absent or too low for efficient targeting $(11,14)$. Interestingly, most somatostatin receptor-negative insulinomas do express the glucagonlike peptide-1 receptor (GLP-1R), often in high density (11). Several studies have demonstrated the feasibility of targeting GLP-1R with radiolabeled exendin-4 $(6,11,15-17)$, a synthetic, metabolically more stable peptide analog of GLP-1. However, a critical concern remains the high kidney uptake. This concern is 2-fold: as the tail of the pancreas is near the left kidney, localization and quantification of radiolabeled exendin- 4 in the tail is hampered by signal spillover from the left kidney, and the high kidney uptake limits the applicability of exendin-4 for PRRT.

One approach to reducing renal accumulation of radiolabeled peptides is to infuse succinylated gelatin (Gelofusine; Braun) (18). In preclinical animal models, Gelofusine efficiently reduced the renal accumulation of radiolabeled peptides, including exendin $(19,20)$. The goal of this study was to investigate whether Gelofusine can also reduce the renal uptake of ${ }^{111}$ In-labeled exendin-4 in humans and to perform dosimetric calculations to estimate the impact of Gelofusine on kidney, islet, and tumor radiation dose. With these data, we aimed to estimate the maximum radiation dose that can be safely administered without causing radiotoxicity to normal organs when labeled with ${ }^{177} \mathrm{Lu}$. 


\section{MATERIALS AND METHODS}

\section{Subjects}

Eleven healthy volunteers were recruited for this study (5 men, 6 women), of whom 1 female participant withdrew consent. Participants were eligible if older than $18 \mathrm{y}$, with no more than $7 \%$ glycated hemoglobin and normal renal function (creatinine clearance $>90 \mathrm{~mL} /$ min by the Cockcroft-Gault formula). Exclusion criteria included heart or liver failure, hypertension, pregnancy, lactation, known hypersensitivity to Gelofusine or exendin, or a history of anaphylaxis. Written informed consent was obtained from all study participants in accordance with provisions of the Declaration of Helsinki, and the study was approved by the Institutional Ethics Review Board of the Radboud University Medical Center (ClinicalTrials.gov number NCT02541734). Patient characteristics are listed in Supplemental Table 1 (supplemental materials are available at http://jnm.snmjournals.org).

\section{Study Design}

To study the effect of Gelofusine on the renal accumulation of ${ }^{111}$ In-labeled exendin-4, we performed a controlled crossover trial with 2 arms (Fig. 1). For each arm, 2 series of measurements were obtained: ${ }^{111}$ In-labeled $\left[\mathrm{Lys}^{40}(\mathrm{Ahx}-\mathrm{DTPA})\right]$ exendin-4 SPECT/CT with saline $(0.9 \% \mathrm{NaCl})$ (saline) and ${ }^{111} \mathrm{In}$-labeled exendin-4 SPECT/CT with Gelofusine.

${ }^{111}$ In-labeled $\left[\operatorname{Lys}^{40}(\mathrm{Ahx}-\mathrm{DTPA})\right]$ exendin-4, where DTPA is diethylenetriaminepentaacetic acid, was prepared as previously described (16). The day before imaging, participants were asked to drink at least $2 \mathrm{~L}$ of water and avoid the consumption of coffee, cola, and tea. Subjects fasted for at least $4 \mathrm{~h}$ before ${ }^{111} \mathrm{In}$-labeled $\left[\mathrm{Lys}^{40}\right.$ (Ahx-DTPA)]exendin-4 injection $(50 \mathrm{MBq})$. Blood pressure and heart rate were monitored before and $5,15,30,60,120,180$, and $240 \mathrm{~min}$ after infusion. Blood samples were obtained from an intravenous catheter in the contralateral arm at 2, 10, 20, and $40 \mathrm{~min}$ before and at 1, 2, 3, and $24 \mathrm{~h}$ after injection. Urine was collected for $24 \mathrm{~h}$ at two 3 -h intervals followed by a 6-h and a 12-h interval. Radioactivity in blood and urine was measured with an automatic $\gamma$-counter (2480-Wizard; Perkin-Elmer). Blood glucose was measured before and $15 \mathrm{~min}$ after tracer injection.

\section{SPECT Acquisition and Reconstruction}

At $24 \mathrm{~h}$ after tracer injection, SPECT images were acquired using an integrated SPECT/CT scanner (Symbia T16; Siemens Healthcare) and reconstructed as described previously (16). A fixed radius of rotation $(25 \mathrm{~cm})$ and bed height $(12 \mathrm{~cm})$ were applied.

\section{Image Analysis}

To determine the kidney uptake, a threshold-based volume of interest (VOI) was drawn around the kidneys in the saline scan, including $80 \%$ of the most radioactive voxels, using the Inveon Research Workplace software (Siemens Inc.). This same VOI was used to determine the kidney uptake in the Gelofusine scan.
To determine pancreatic uptake, 2 small spheric VOIs were drawn in the pancreas as described previously (16). The pancreatic uptake was presented as the mean of the counts $/ \mathrm{mm}^{3}$ of both VOIs. For background correction, identical VOIs were placed in the liver.

\section{Dosimetric Calculations}

To investigate the potential of ${ }^{177} \mathrm{Lu}$-labeled exendin for insulinoma treatment, dosimetric calculations were performed using our macroscale and small-scale dosimetry model (21). The time-integrated activity coefficients (TIAC) and dose rate $\mathrm{S}$ values of the kidneys, pancreas, islets, and remainder of the body were determined previously (21). These values were corrected for the longer half-life of ${ }^{177} \mathrm{Lu}$ than of ${ }^{111} \mathrm{In}$, assuming similar biodistribution of the ${ }^{111} \mathrm{In}$ - and ${ }^{177} \mathrm{Lu}$-labeled compounds. As previously described (21), for the macroscale and small-scale dosimetry model the average TIAC of 5 healthy volunteers was used as input. For simulation of humans with high or low kidney uptake, the maximum and minimum TIAC of these 5 volunteers was used, respectively.

To obtain the TIAC of insulinomas, we analyzed ${ }^{111}$ In-DOTA-exendin4 planar whole-body scintigraphic images acquired at various time-points after injection for 5 patients with an insulinoma (Supplemental Table 2). These scans were obtained at the university hospital in Basel, Switzerland, and have previously been published $(11,22)$. Regions of interest were manually drawn around the insulinomas on both the anterior and the posterior scans. Corresponding background regions were placed close to the regions of interest for background correction. Activity concentrations were evaluated assuming a uniform distribution in the tumor. The geometric mean obtained from the anterior and posterior views was corrected for attenuation using the conjugate view method. The total whole-body counts (geometric mean between anterior and posterior views) acquired 20 min after injection of the tracer was set as $100 \%$ injected activity, providing a calibration factor between counts and activity for planar recordings. The activity concentrations were expressed as percentage injected activity and corrected for the longer half-life of ${ }^{177} \mathrm{Lu}$. All dosimetric evaluations were done with OLINDA/EXM software, version 1.1 (23), using the whole-body adult female and male models as well as the weighting factors recommended by publication 60 of the International Commission on Radiological Protection (23). The area under the curve was determined assuming only physical decay after the last measured time point. The insulinoma absorbed dose was calculated using the sphere model of OLINDA/EXM (24). Tumor mass was estimated on the basis of diameter measurements, assuming a tumor density of $1 \mathrm{~g} / \mathrm{cm}^{3}$.

The maximum absorbed dose that could safely be delivered to the insulinoma without causing radiotoxicity to normal organs was defined as the dose that would lead to a maximum absorbed kidney dose of 23 Gy (25).

\section{Statistical Analysis}

Statistical analyses were performed using SPSS, version 22 (SPSS). Results were presented as mean \pm SD. $P$ values of less than 0.05 were considered statistically significant. An a priori power analysis was

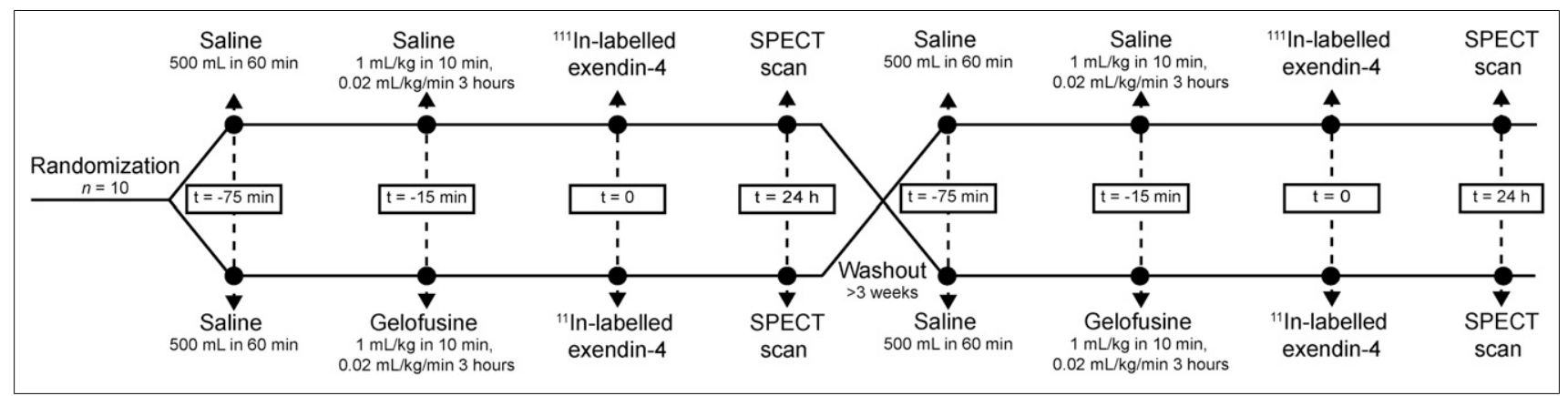

FIGURE 1. Time line of crossover trial. 
performed for the primary outcome measure (kidney uptake) based on our previous preclinical and clinical studies using ${ }^{111}$ In-labeled exendin-4 $(16,19,26)$. A decrease in anticipated kidney uptake of $10 \%$ was considered clinically relevant. Two groups of 10 participants each would allow a statistical power of 0.80 (paired data, $\mathrm{SD}=10 \%, \alpha=$ $0.05, \rho=0.5$ ). After data collection, normality of the distributions was assessed using the Shapiro-Wilk test. Between-group differences were analyzed by ANOVA. Differences in organ uptake between saline and Gelofusine scans were analyzed using general-linear-model univariate repeated-measures procedures, with Gelofusine and saline as the withinsubject factor and the sequence of the scans as the between-subject factor. The activity curves of the blood and urine were analyzed with mixedmodel ANOVA with Bonferroni post hoc testing.

\section{RESULTS}

\section{Gelofusine Significantly Reduces Kidney Uptake of Radiolabeled Exendin-4}

To determine whether coadministration of Gelofusine could significantly reduce the kidney uptake of ${ }^{111}$ In-labeled exendin-4, 10 healthy subjects were assigned to 1 of 2 groups (saline-Gelofusine or Gelofusine-saline) in a crossover design. Relevant parameters such as creatinine clearance and glycated hemoglobin did not significantly differ between the groups $(P=0.4$ and $P=0.6$, respectively). The infusion of saline, Gelofusine, and exendin did not cause any side effects.

Representative ${ }^{111}$ In-labeled exendin-4 SPECT/CT scans of the kidneys and pancreas are depicted in Figures 2A, 2B, 2D, and 2E, with 3dimensional projections shown in Figures $2 \mathrm{C}$ and $2 \mathrm{~F}$. In all subjects, infusion of Gelofusine reduced the renal uptake of ${ }^{111}$ In-labeled exendin-4 (Fig. 2G) $\left(F_{1,8}=136.9, P=0.000003\right)$, with a mean reduction of $18.1 \% \pm 4.2 \%$, whereas the activity concentration in the pancreas remained unchanged $\left(F_{1,8}=1.196, P=0.3\right)$ (Fig. $\left.2 \mathrm{H}\right)$. In 3 of 10 subjects, the kidney uptake was reduced to such an extent that the pancreatic tail could be more clearly discriminated from the kidney signal (Figs. 2C and 2F). There was no evidence that the sequence of saline or Gelofusine infusion had an effect on this outcome $\left(F_{1,8}=\right.$ $0.102, P=0.8)$. Parallel to the reduction in kidney uptake, we observed a significantly higher cumulative excretion of ${ }^{111} \mathrm{In}$-labeled exendin-4 in the urine after Gelofusine infusion (Fig. 2I). The blood clearance of ${ }^{111}$ In-labeled exendin- 4 was significantly reduced in the first $10 \mathrm{~min}$ when coinfused with Gelofusine (Fig. 2J), but pancreatic uptake was not affected.
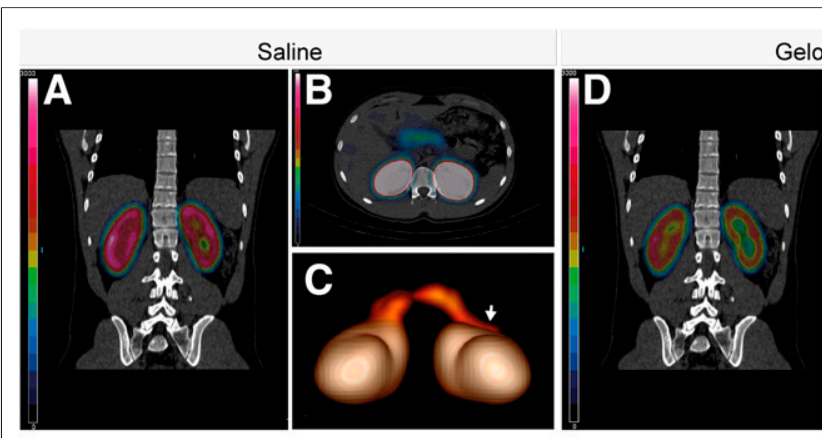

Gelofusine

G

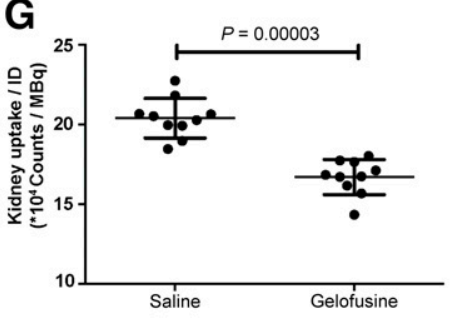

I

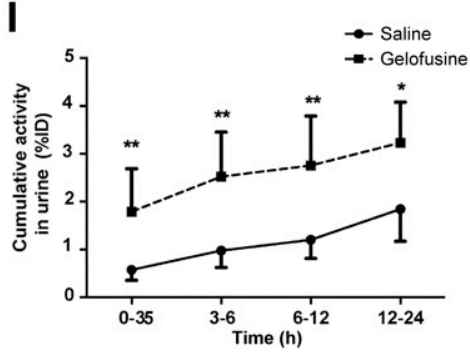

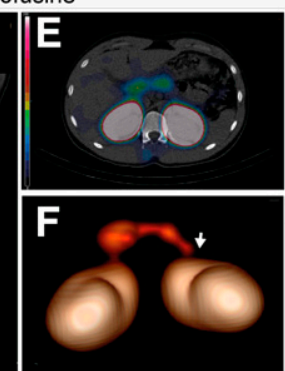

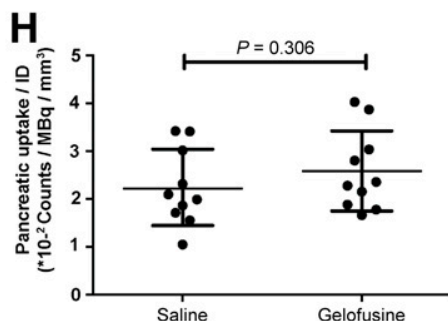

J

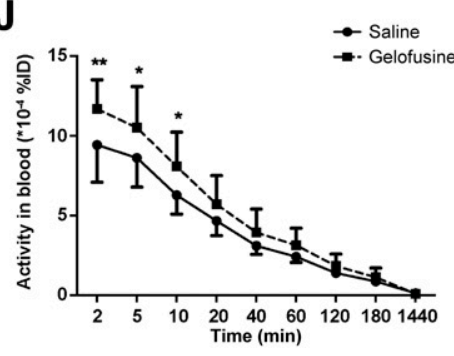

FIGURE 2. Quantitative analysis of kidney and pancreatic uptake of ${ }^{111} \mathrm{In}$-labeled exendin-4 in SPECT/CT scans. SPECT/CT images (A, B, D, and E) were obtained $24 \mathrm{~h}$ after ${ }^{111} \mathrm{In}$-exendin injection, coinfused with saline $(A$ and $B)$ or Gelofusine ( $D$ and $E)$. In 3 of 10 patients, 3-dimensional projections of SPECT images ( $\mathrm{C}$ and $\mathrm{F})$ demonstrated that pancreas could be more clearly discriminated from kidney signal after Gelofusine injection (arrows) (F). Kidney uptake of ${ }^{111}$ Inlabeled exendin-4 was significantly reduced after administration of Gelofusine (G), whereas activity concentration in pancreas remained same $(\mathrm{H})$. Clearance of ${ }^{111} \mathrm{In}$-labeled exendin-4 was determined in urine (I) and in blood $(\mathrm{J}) .{ }^{\star} P<0.05 .{ }^{\star \star} P<0.01$. ID $=$ injected dose.

\section{Gelofusine Potentiates Higher Exendin Injection Dose}

As the GLP-1 receptor is abundantly expressed in most insulinomas (14), we hypothesized that exendin may be a suitable candidate for PRRT when labeled with ${ }^{177} \mathrm{Lu}$. To estimate the radiation dose that can be delivered to insulinomas using ${ }^{177} \mathrm{Lu}$-labeled exendin, we determined the TIAC of benign and malignant insulinomas based on planar scintigraphic images of 5 patients after injection with either ${ }^{111}$ In-DOTA- or ${ }^{111}$ In-DTPA-exendin-4 (Supplemental Table 2 and Table 1). ${ }^{111}$ In-labeled exendin-4 was converted to ${ }^{177} \mathrm{Lu}$-labeled exendin-4 under the assumption that the biodistribution pattern of the tracer is not affected by chelator or radiometal cation type. On the basis of the previously reported TIAC of the kidneys, pancreas, and islets (21), we could estimate the tumor and islet radiation dose using our macroscale and small-scale dosimetry model (21).

Since the kidneys are the dose-limiting organs in the case of exendin (21), we calculated the highest activity that could be administered and the concomitant absorbed insulinoma and islet doses that would result in the maximum allowed absorbed kidney dose of 23 Gy (25) for a male or female with a low or high kidney uptake (Table 1). For simulation with high or low kidney uptake, the maximum and minimum observed TIAC of the kidneys was used, respectively. In the most favorable situation (male with a low kidney uptake), the maximum activity that could be administered was $1.79 \mathrm{GBq}$, resulting in an absorbed insulinoma dose ranging from 54.2 Gy to 127.8 Gy. In the least favorable situation 
TABLE 1

Dosimetry Calculations for ${ }^{177}$ Lu-Labeled Exendin

\begin{tabular}{|c|c|c|c|c|c|c|c|c|c|c|c|c|c|c|c|c|c|c|c|c|}
\hline \multirow{3}{*}{ Parameter } & \multicolumn{10}{|c|}{ Low kidney uptake } & \multicolumn{10}{|c|}{ High kidney uptake } \\
\hline & \multicolumn{2}{|c|}{ Patient 1} & \multicolumn{2}{|c|}{ Patient 2} & \multicolumn{2}{|c|}{ Patient 3} & \multicolumn{2}{|c|}{ Patient 4} & \multicolumn{2}{|c|}{ Patient 5} & \multicolumn{2}{|c|}{ Patient 1} & \multicolumn{2}{|c|}{ Patient 2} & \multicolumn{2}{|c|}{ Patient 3} & \multicolumn{2}{|c|}{ Patient 4} & \multicolumn{2}{|c|}{ Patient 5} \\
\hline & M & $\mathrm{F}$ & M & $\mathrm{F}$ & M & $\mathrm{F}$ & M & $\mathrm{F}$ & M & $\mathrm{F}$ & M & $\mathrm{F}$ & M & $\mathrm{F}$ & M & $\mathrm{F}$ & M & $\mathrm{F}$ & M & $\mathrm{F}$ \\
\hline TIAC insulinoma (h) & 0.44 & 0.44 & 0.81 & 0.81 & 0.65 & 0.65 & 0.62 & 0.62 & 0.96 & 0.96 & 0.44 & 0.44 & 0.81 & 0.81 & 0.65 & 0.65 & 0.62 & 0.62 & 0.96 & 0.96 \\
\hline Kidney dose (Gy) & 23 & 23 & 23 & 23 & 23 & 23 & 23 & 23 & 23 & 23 & 23 & 23 & 23 & 23 & 23 & 23 & 23 & 23 & 23 & 23 \\
\hline \multicolumn{21}{|l|}{ Without Gelofusine } \\
\hline Max. inj. activity (GBq) & 1.79 & 1.64 & 1.79 & 1.64 & 1.79 & 1.64 & 1.79 & 1.64 & 1.79 & 1.64 & 1.12 & 1.04 & 1.12 & 1.04 & 1.12 & 1.04 & 1.12 & 1.04 & 1.12 & 1.04 \\
\hline Insulinoma dose (Gy) & 127.8 & 117.4 & 54.2 & 48.0 & 90 & 82.7 & 78.5 & 72.2 & 112.2 & 103.1 & 80.2 & 74.1 & 32.9 & 30.3 & 56.5 & 52.2 & 49.3 & 45.5 & 70.7 & 65.1 \\
\hline Islet dose (Gy) & 4.0 & 4.8 & 4.0 & 4.8 & 4.0 & 4.8 & 4.0 & 4.8 & 4.0 & 4.8 & 2.6 & 3.0 & 2.6 & 3.0 & 2.6 & 3.0 & 2.6 & 3.0 & 2.6 & 3.0 \\
\hline \multicolumn{21}{|l|}{ With Gelofusine } \\
\hline Max. inj. activity (GBq) & 2.18 & 2.01 & 2.18 & 2.01 & 2.18 & 2.01 & 2.18 & 2.01 & 2.18 & 2.01 & 1.38 & 1.26 & 1.38 & 1.26 & 1.38 & 1.26 & 1.38 & 1.26 & 1.38 & 1.26 \\
\hline Insulinoma dose (Gy) & 156.1 & 143.6 & 63.8 & 58.7 & 109.9 & 101.1 & 95.9 & 88.2 & 137.0 & 126.0 & 98.5 & 90.2 & 40.2 & 36.9 & 69.3 & 63.5 & 60.5 & 55.4 & 86.4 & 79.2 \\
\hline Islet dose (Gy) & 4.9 & 5.8 & 4.9 & 5.8 & 4.9 & 5.8 & 4.9 & 5.8 & 4.9 & 5.8 & 3.1 & 3.7 & 3.1 & 3.7 & 3.1 & 3.7 & 3.1 & 3.7 & 3.1 & 3.7 \\
\hline
\end{tabular}

(female with a high kidney uptake), the injection activity could be only $1.04 \mathrm{GBq}$, with a concomitant absorbed dose ranging from $30.3 \mathrm{~Gy}$ to 74.1 Gy (Table 1). The maximum absorbed islet radiation dose (diameter islets, $400 \mu \mathrm{m}$ ) varied between 2.6 and $4.8 \mathrm{~Gy}$.

Because Gelofusine effectively reduced the kidney uptake of ${ }^{111} \mathrm{In}-$ labeled exendin, protection of the kidneys allows a higher maximum injection activity. If we assume that the observed reduction would be similar for ${ }^{177} \mathrm{Lu}$-labeled exendin and that Gelofusine would not interfere with the activity accumulation in other tissues, the maximum activity that could be injected would be $2.18 \mathrm{GBq}$ (Table 1). In these cases, the highest estimated absorbed insulinoma dose would be 156.1 Gy and the maximum islet dose would remain below 5.9 Gy (Table 1).

\section{DISCUSSION}

In line with previous results in rodents $(19,20,26)$, we now demonstrate that Gelofusine can substantially reduce the renal accumulation of ${ }^{111} \mathrm{In}$-labeled [ $\left.\mathrm{Lys}^{40}(\mathrm{Ahx}-\mathrm{DTPA})\right]$ exendin- 4 in humans. With a mean reduction of $18.1 \%$, the kidney uptake becomes sufficiently low to enable better discrimination of the pancreatic tail from the kidney signal. This reduction not only allows more accurate analyses of GLP-1R expression in the tail region of the pancreas but also potentiates the safe delivery of a higher radiation dose to GLP-1R-positive tumors in the case of PRRT.

PRRT is considered an alternative therapy in cases of unresectable or multifocal insulinomas with metastasis. Even if complete remission were not feasible, this therapy could improve progression-free and overall survival, normalize blood glucose levels, and prevent recurrent episodes of severe hypoglycemia. Our preliminary estimation of the insulinoma absorbed dose predicts that a dose between 30.3 and 127.8 Gy can be safely delivered to the insulinoma-an absorbed dose range shown to induce tumor shrinkage in pancreatic neuroendocrine tumors treated with ${ }^{177} \mathrm{Lu}$-labeled DOTATATE (27). Here, we show this dose can be further enhanced when Gelofusine is used to protect the kidneys.

An important factor often neglected is the islet absorbed dose. Reports usually present the pancreatic absorbed dose (28), but since this approximation assumes homogeneous tracer distribution, this dose underestimates the radiation dose to the islets of Langerhans. Using our dedicated macroscale and small-scale dosimetry model
(21), we estimated that the islet absorbed dose for islets with a diameter of $400 \mu \mathrm{m}$ would be below $5.9 \mathrm{~Gy}$. As most human islets have a diameter between 50 and $100 \mu \mathrm{m}$ (29), the islet absorbed dose for most islets would be lower. Although the exact radiation dose causing islet damage is unknown, we do know that $4.6 \%$ of the children who received a pancreatic dose of $4.4 \pm 8.7$ Gy with radiation therapy developed diabetes by the age of $45 \mathrm{y}(30)$. As most insulinoma patients are adults, we believe that the risk of developing diabetes is sufficiently low, especially considering the potentially life-threatening symptoms if the insulinomas cannot be surgically removed.

The only other study, to our knowledge (by Velikyan et al. (28)), that performed dosimetric estimations for ${ }^{177} \mathrm{Lu}$-labeled exendin reported an absorbed insulinoma dose of $0.70 \mathrm{mGy} / \mathrm{MBq}$, with a maximum administrable activity of $3.7 \mathrm{GBq}$. This finding is in marked contrast to our observation that the insulinoma dose varies between 29.3 and 71.6 $\mathrm{mGy} / \mathrm{MBq}$, with a maximum administrable activity between 1.04 and $1.79 \mathrm{GBq}$. The discrepancy between the two studies may be explained, at least to some extent, by methodologic differences.

First, the reported TIACs determined by Velikyan et al. (28) were based on rat ${ }^{177} \mathrm{Lu}$-DO3A-VS-Cys40-exendin-4 biodistribution studies that were extrapolated to the human setting, and on one ${ }^{68} \mathrm{Ga}-\mathrm{DO} 3 \mathrm{~A}-$ VS-Cys40-exendin-4 PET/CT scan in a patient for the quantification of the insulinoma dose. However, it has been reported for other radiolabels that extrapolated rat data underestimate the TIAC of the kidneys by a factor of 1.8 (31), which could explain why Velikyan et al. reported a higher tolerated activity dose. Also, other time points have been used to estimate the TIAC. In our case, the last planar scintigraphic image was taken either 70 or $120 \mathrm{~h}$ after injection, whereas Velikyan et al. performed rat biodistribution studies up to $336 \mathrm{~h}$ after injection (28). After the last time points, both studies assumed that further decline in radioactivity occurs because of physical decay without further biologic clearance. Therefore, our TIAC may even be overestimated, underestimating the radioactivity dose that can safely be administered.

Second, the chelator and radiolabel vary between the two studies. For the dosimetric calculations in our study, the data were corrected only for the longer half-life of ${ }^{177} \mathrm{Lu}$. On the basis of studies that directly compared the biodistribution characteristics of peptides functionalized with different chelators and labeled with various isotopes, we know that both 
the chelator (32-34) and the radiolabel $(34,35)$ can influence the receptor affinity and biodistribution features of the tracer, as may explain the differences between the findings reported in the two studies. However, since our preclinical studies demonstrate that the use of chelators such as DTPA and DOTA, a potential chelator for ${ }^{177} \mathrm{Lu}(36)$, does not affect the biodistribution of exendin, we assumed similar biodistribution characteristics between DOTA- and DTPA-exendin tracers in humans.

Third, our estimation of the absorbed insulinoma dose was based on whole-body planar images, whereas the results of Velikyan et al. were based on a ${ }^{68} \mathrm{Ga}$-exendin PET scan (28). Because planar imaging detects radiation emanating from activity at all depths of the subject, the tumor, as well as surrounding background tissue, contributes to the detected counts. Although the background uptake of radiolabeled exendin is low, we potentially overestimated the absorbed insulinoma dose.

The predicted insulinoma absorbed dose we describe here closely resembles the dose range previously shown to successfully treat pancreatic neuroendocrine tumors with ${ }^{177} \mathrm{Lu}$-labeled DOTATATE (27). These initial results suggest that GLP-1R targeting may be an interesting treatment strategy for patients with unresectable or metastatic insulinomas that are somatostatin receptor-negative, especially since most somatostatin receptor-negative insulinomas do express GLP-1R (11). However, given the high interpatient variability in GLP-1R expression $(11,14)$, insulinoma size, and kidney uptake $(21)$, we do recognize that the absorbed tumor and organ doses may vary considerably between individuals and that studies with low-dose ${ }^{177} \mathrm{Lu}$-labeled exendin- 4 are warranted to extend these preliminary observations.

\section{CONCLUSION}

Gelofusine substantially reduces the renal accumulation of radiolabeled exendin- 4 in humans, potentiating the safe administration of clinically relevant injection activities for exendin-based PRRT.

\section{DISCLOSURE}

This work was supported by BetaCure (FP7/2014-2018, grant agreement 602812) and INNODIA (IMI2-JU, grant agreement 115797). Martin Béhé and Martin Gotthardt are an inventor and holder of the patent "Invention Affecting GLP-1 and Exendin" (Philipps-Universität Marburg, June 17, 2009). No other potential conflict of interest relevant to this article was reported.

\section{REFERENCES}

1. Grant CS. Insulinoma. Best Pract Res Clin Gastroenterol. 2005;19:783-798.

2. Mehrabi A, Fischer L, Hafezi M, et al. A systematic review of localization, surgical treatment options and outcome of insulinoma. Pancreas. 2012;41:1363-1363.

3. Placzkowski KA, Vella A, Thompson GB, et al. Secular trends in the presentation and management of functioning insulinoma at the Mayo Clinic, 1987-2007. J Clin Endocrinol Metab. 2009;94:1069-1073.

4. Maecke HR, Hofmann M, Haberkorn U. ${ }^{68}$ Ga-labeled peptides in tumor imaging. J Nucl Med. 2005;46(suppl):172S-178S.

5. Frilling A, Sotiropoulos GC, Radtke A, et al. The impact of ${ }^{68} \mathrm{Ga}$-DOTATOC positron emission tomography/computed tomography on the multimodal management of patients with neuroendocrine tumors. Ann Surg. 2010;252:850-856.

6. Eriksson O, Velikyan I, Selvaraju RK, et al. Detection of metastatic insulinoma by positron emission tomography with $\left[{ }^{68} \mathrm{Ga}\right]$ exendin-4: a case report. J Clin Endocrinol Metab. 2014;99:1519-1524.

7. Christ E, Wild D, Ederer S, et al. Glucagon-like peptide-1 receptor imaging for the localisation of insulinomas: a prospective multicentre imaging study. Lancet Diabetes Endocrinol. 2013;1:115-122.

8. Brabander T, Teunissen JJM, Van Eijck CHJ, et al. Peptide receptor radionuclide therapy of neuroendocrine tumours. Best Pract Res Clin Endocrinol Metab. 2016;30:103-114.

9. Imhof A, Brunner P, Marincek N, et al. Response, survival, and long-term toxicity after therapy with the radiolabeled somatostatin analogue $\left[{ }^{90} \mathrm{Y}\right.$-DOTA]TOC in metastasized neuroendocrine cancers. J Clin Oncol. 2011;29:2416-2423.
10. Kwekkeboom DJ, De Herder WW, Kam BL, et al. Treatment with the radiolabeled somatostatin analog $\left[{ }^{177} \mathrm{Lu}-\mathrm{DOTA}{ }^{0}, \mathrm{Tyr}^{3}\right]$ octreotate: toxicity, efficacy, and survival. J Clin Oncol. 2008;26:2124-2130.

11. Wild D, Christ E, Caplin ME, et al. Glucagon-like peptide-1 versus somatostatin receptor targeting reveals 2 distinct forms of malignant insulinomas. $\mathrm{J} \mathrm{Nucl} \mathrm{Med}$. 2011;52:1073-1078.

12. Ezziddin S, Khalaf F, Vanezi M, et al. Outcome of peptide receptor radionuclide therapy with ${ }^{177} \mathrm{Lu}$-octreotate in advanced grade $1 / 2$ pancreatic neuroendocrine tumours. Eur J Nucl Med Mol Imaging. 2014;41:925-933.

13. Kam BLR, Teunissen JJM, Krenning EP, et al. Lutetium-labelled peptides for therapy of neuroendocrine tumours. Eur J Nucl Med Mol Imaging. 2012;39(suppl 1):S103-S112.

14. Reubi JC, Waser B. Concomitant expression of several peptide receptors in neuroendocrine tumours: molecular basis for in vivo multireceptor tumour targeting. Eur J Nucl Med Mol Imaging. 2003;30:781-793.

15. Brom M, Joosten L, Frielink C, Boerman O, Gotthardt M. ${ }^{111}$ In-exendin uptake in the pancreas correlates with the $\beta$-cell mass and not with the $\alpha$-cell mass. Diabetes. 2015;64:1324-1328.

16. Brom M, Woliner-van der Weg W, Joosten L, et al. Non-invasive quantification of the beta cell mass by SPECT with ${ }^{111}$ In-labelled exendin. Diabetologia. 2014;57:950-959.

17. Wicki A, Wild D, Storch D, et al. [Lys ${ }^{40}\left(\right.$ Ahx-DTPA- $\left.\left.{ }^{111} \mathrm{In}\right) \mathrm{NH}_{2}\right]$-exendin-4 is a highly efficient radiotherapeutic for glucagon-like peptide-1 receptor-targeted therapy for insulinoma. Clin Cancer Res. 2007;13:3696-3705.

18. Veldman BAJ, Schepkens HLE, Vervoort G, Klasen I, Wetzels JFM. Low concentrations of intravenous polygelines promote low-molecular weight proteinuria. Eur J Clin Invest. 2003;33:962-968.

19. Vegt E, Eek A, Oyen WJG, de Jong M, Gotthardt M, Boerman OC. Albuminderived peptides efficiently reduce renal uptake of radiolabelled peptides. Eur J Nucl Med Mol Imaging. 2010;37:226-234.

20. Vegt E, van Eerd JEM, Eek A, et al. Reducing renal uptake of radiolabeled peptides using albumin fragments. J Nucl Med. 2008;49:1506-1511.

21. van der Kroon I, Woliner-van der Weg W, Brom M, et al. Whole organ and islet of Langerhans dosimetry for calculation of absorbed doses resulting from imaging with radiolabeled exendin. Sci Rep. 2017;7:39800.

22. Christ E, Wild D, Forrer F, et al. Glucagon-like peptide-1 receptor imaging for localization of insulinomas. J Clin Endocrinol Metab. 2009;94:4398-4405.

23. Stabin MG, Sparks RB, Crowe E. OLINDA/EXM: the second-generation personal computer software for internal dose assessment in nuclear medicine. $\mathrm{J} \mathrm{Nucl}$ Med. 2005;46:1023-1027.

24. Stabin MG, Komjnenberg MW. Re-evaluation of absorbed fractions for photons and electrons in spheres of various sizes. J Nucl Med. 2000;41:149-160.

25. Dawson LA, Kavanagh BD, Paulino AC, et al. Radiation-associated kidney injury. Int J Radiat Oncol Biol Phys. 2010;76(suppl):S108-S115.

26. Gotthardt M, van Eerd-Vismale J, Oyen WJG, et al. Indication for different mechanisms of kidney uptake of radiolabeled peptides. J Nucl Med. 2007;48:596-601.

27. Ilan E, Sandstrom M, Wassberg C, et al. Dose response of pancreatic neuroendocrine tumors treated with peptide receptor radionuclide therapy using ${ }^{177} \mathrm{Lu}$ DOTATATE. J Nucl Med. 2015;56:177-182.

28. Velikyan I, Bulenga TN, Selvaraju R, et al. Dosimetry of $\left[{ }^{177} \mathrm{Lu}\right]$-do3a-vs-cys ${ }^{40}$ exendin-4: impact on the feasibility of insulinoma internal radiotherapy. Am J Nucl Med Mol Imaging. 2015;5:109-126.

29. Kilimnik G, Jo J, Periwal V, Zielinski MC, Hara M. Quantification of islet size and architecture. Islets. 2012;4:167-172.

30. de Vathaire F, El-Fayech C, Ben Ayed FF, et al. Radiation dose to the pancreas and risk of diabetes mellitus in childhood cancer survivors: a retrospective cohort study. Lancet Oncol. 2012;13:1002-1010.

31. Selvaraju RK, Bulenga TN, Espes D, Lubberink M, Sörensen J. Dosimetry of [ $\left[{ }^{68} \mathrm{Ga}\right]-$ Ga-D03A-VS-Cys ${ }^{40}$-exendin- 4 in rodents, pigs, non-human primates and human: repeated scanning in human is possible. Am J Nucl Med Mol Imaging. 2015;5:259-269.

32. Fani M, Del Pozzo L, Abiraj K, et al. PET of somatostatin receptor-positive tumors using ${ }^{64} \mathrm{Cu}$ - and ${ }^{68} \mathrm{Ga}$-somatostatin antagonists: the chelate makes the difference. J Nucl Med. 2011;52:1110-1118.

33. Kwekkeboom DJ, Kooij PP, Bakker WH, Mäcke HR, Krenning EP. Comparison of ${ }^{111} \mathrm{In}$-DOTA-Tyr ${ }^{3}$-octreotide and ${ }^{111}$ In-DTPA-octreotide in the same patients: biodistribution, kinetics, organ and tumor uptake. J Nucl Med. 1999;40:762-767.

34. Reubi JC, Schär J-C, Waser B, et al. Affinity profiles for human somatostatin receptor subtypes SST1-SST5 of somatostatin radiotracers selected for scintigraphic and radiotherapeutic use. Eur J Nucl Med. 2000;27:273-282.

35. Bauman A, Valverde IE, Fischer CA, Vomstein S, Mindt TL. Development of ${ }^{68} \mathrm{Ga}$-and ${ }^{89} \mathrm{Zr}$-labeled exendin-4 as potential radiotracers for the imaging of insulinomas by PET. J Nucl Med. 2015;56:1569-1574.

36. Stimmel JB, Kull FC. Samarium-153 and lutetium-177 chelation properties of selected macrocyclic and acyclic ligands. Nucl Med Biol. 1998;25:117-125. 